\title{
Preface: special issue on the annual international conference on combinatorial optimization and applications (COCOA)
}

\section{Xiaofeng Gao ${ }^{1}$}

Accepted: 6 October 2021 / Published online: 21 October 2021

(c) The Author(s), under exclusive licence to Springer Science+Business Media, LLC, part of Springer Nature 2021

This special issue of Journal of Combinatorial Optimization consists of selected papers that were presented at the 11th Annual International Conference on Combinatorial Optimization and Applications (COCOA), taken place in Shanghai, People's Republic of China, during December 16-18, 2017.

COCOA 2017 provided a forum for researchers working in the area of theoretical computer science and combinatorics. The technical program of the conference included 59 regular papers selected by the Program Committee from 145 full submissions received in response to the call for papers. Each submission was peer reviewed by at least 3 , and on the average 3.8, Program Committee members or external reviewers. The topics cover most aspects of theoretical computer science and combinatorics related to computing, including classic combinatorial optimization, geometric optimization, complexity and data structures, graph theory, etc.

We would like to thank the authors for contributing their works, and program committee members and external reviewers for volunteering their time to review the papers.

Publisher's Note Springer Nature remains neutral with regard to jurisdictional claims in published maps and institutional affiliations.

Xiaofeng Gao

gao-xf@cs.sjtu.edu.cn

1 Department of Computer Science and Engineering, Shanghai Jiao Tong University, Shanghai, China 